\title{
Professional Clinical Photography to Assist Patients with Pectus Carinatum
}

\author{
John A. Yeats
}

A photographic clinical role has been realised in our institution in the care of patients with Pectus Carinatum. A series of clinical photographs along with $3 D$ imaging are now presented by our photographers to each patient in clinic to demonstrate changes over time in the size and shape of their chest wall defects associated with this condition. This interaction has increased patient compliance for treatment and subsequently reinforces the relevance and importance of clinical photographers in patient care.

OPEN ACCESS

\section{Keywords}

Pectus carinatum, clinical photography, 3D photography, orthotics

\section{Introduction}

Pectus carinatum is a disorder of the thoracic skeleton, described by Krummel (1998) as a "structural deformity of the sternum in which a sternal protrusion occurs." This protrusion is the result of cartilage overgrowth. It is estimated that pectus carinatum accounts for approximately 5\% of all chest wall deformities (Goretsky et al. 2004), with the disorder more commonly found in males than females (Krummel 1998).

There are a number of patterns of pectus carinatum that can occur depending upon the location of the protrusion within the sternum. Protrusions of the middle and lower portions of the sternum are the most common type of pectus carinatum (Nuchtern et al. 2012), and are known as the 'chicken breast' or 'keel chest' abnormality due to the deformity's appearance. Protrusions of the upper portion of the sternum are commonly referred to as 'Pouter pigeon deformity' (Goretsky et al. 2004). The general term 'pigeon chest' is a common name for any form of pectus carinatum.

The use of avian terminology to describe this condition derives from the Latin word for 'keel' - carina. Pectus carinatum literally means 'chest with keel' (Nuchtern et al. 2012). It is noted by Hefti that expressions taken from the animal world, such as those cited above, may 'have very negative connotations' and should be avoided by professional medical staff in their dealings with patients and parents (Nefti 2007).

The majority of cases with pectus carinatum are relatively asymptomatic in physiological terms. However, the nature of the deformity can cause significant psychological distress given the cosmetic (often asymmetrical) appearance of the protruding chest wall. There are particular concerns for adolescent patients in terms of their body image and self-esteem.

Koumbourlis (2015) suggests that patients with pectus carinatum are likely to be affected psychologically as it is difficult to hide a protuberance of the sternum under clothing. Koumbourlis (2015) adds "the poor self-image often leads to social isolation and physical deconditioning that further exacerbates the cardiopulmonary effects of the deformity".

Treatment options for pectus carinatum include surgical intervention to remove cartilage in affected areas, and a more conservative approach using an orthotic brace to actively reshape the chest wall over time. At The Children's Hospital at Westmead, Sydney, New South Wales, Australia, the latter approach is typically adopted given the risk of recurrent protuberances following cartilage excision, as well as scarring and potential blood loss which have also been reported following surgery (Loff et al. 2014). Orthotic brace treatment is also not without its complications with patient compliance for the treatment being the most significant barrier to treatment success. This treatment involves the patients wearing a custom-made orthotic brace made from a cast of the patient's chest.

The brace consists of an aluminum bar, shoulder straps and tightening toggles, with the point of contact with the pectus achieved using a thermoplastic plate, which is cut to size and molded to fit the patient's chest. (Figure 1).

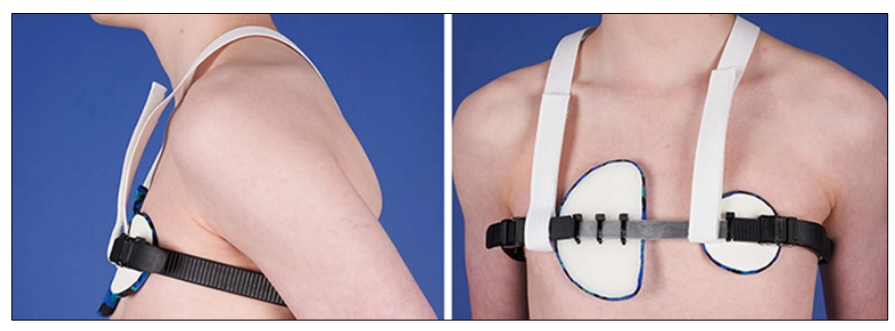

Figure 1. Lateral and anterior views showing newly fitted orthotic brace 
The brace is ideally worn for periods of up to $16-18$ hours per day and is modified and tailored over time depending on the patient's age and rate of improvement. Although the device is worn under clothing in most cases, it is easily noticeable by the patient's peers, particularly in situations such as physical education and school sport, and is thus a source of potential embarrassment for the patient. As a result, a large part of the treatment of pectus carinatum involves education, encouragement and support for patients and families undergoing this treatment.

The clinical photography department has photographed 122 patients (112 male, 10 female) with pectus carinatum over the past 4 years.

The purpose of this paper is to:

a. Describe the clinical photography department's protocol for imaging patients with pectus carinatum,

b. Describe the clinical photography department's involvement in the presentation of photography records to patients and families as a positive reinforcement technique aimed at improving treatment compliance.

\section{Methods}

The clinical photography department's protocol for imaging patients with pectus carinatum consists of a standard clinical photography informed consent process, followed by both conventional photography and $3 \mathrm{~d}$ imaging as described below. As part of the informed consent process, the sequences of images to be captured are explained to the patient and their parent/carer. Given the pediatric nature of our institution, all clinical photography includes the patient's parent/carer being present in the studio when the patient is photographed.

\section{Studio and Camera Equipment}

Studio lighting is provided by $2 \times 1000 \mathrm{~W}$ flash heads (Bowens), and each light is attached with a $3 \mathrm{x}$ 4-foot soft box. One light is triggered by a Pocket Wizard radio control attached to the camera hot shoe. The second flash head is set to slave cell mode. The flash heads are placed at a 45 -degree angle on each anterior flank, equidistant from the patient. A standard cobalt-blue background is used for the imaging. This is consistent with all studio medical photography in our facility. Image capture is completed via a Nikon D3S camera using a 105 Micro Nikkor f2.8 lens.

\section{Settings and Lighting Ratio}

The flash heads are positioned to allow the patient to be photographed at $\mathrm{f} / 11$. A lighting ratio approximating $3: 1$ is employed in order to create a mild shadow effect to enhance any contour aberrations of the anterior thoracic wall. This is the desired outcome. Delivery of the lighting ratio at $f / 11$ is achieved by an inequality of power setting from the flash heads while the distance is kept equal. It is deemed that any more severe lighting ratio may overemphasize the severity of the pectus condition.

\section{Standardized Sequences and Views}

The primary photographic session is conducted at the time of the initial fitting of the orthotic device, and consists of three image sequences:

1. Brace intact, arms by side

2. Brace removed, arms by side

3. Brace removed, arms resting on head

An example from these sequences is shown in Figure 2.
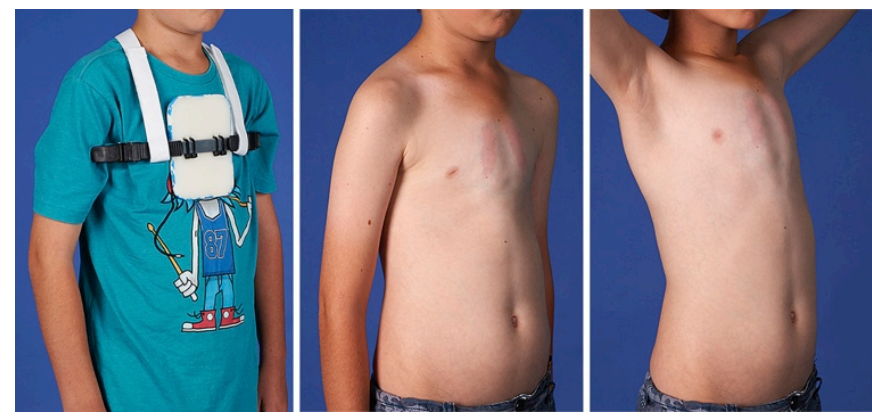

Figure 2. Right oblique view of each of the three photographic sequences

The second, and all subsequent, image sessions consist of the latter two sequences from the initial imaging session. For each of these sequences, the following views are captured:
a. Left lateral
b. Left oblique
c. Anterior
d. Right oblique
e. Right lateral
f. Posterior

An example of these views is shown in Figures 3a,3b,3c.

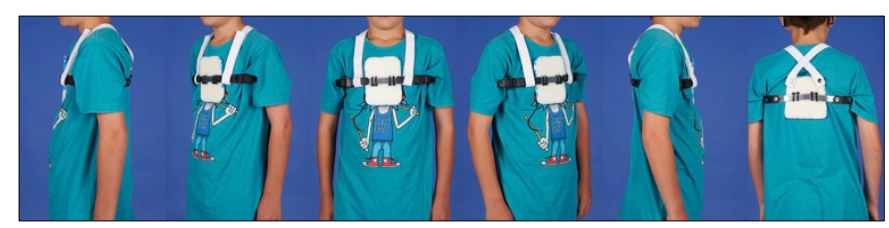

Figure 3a. This serves simply as a visual record of the custom orthotic device

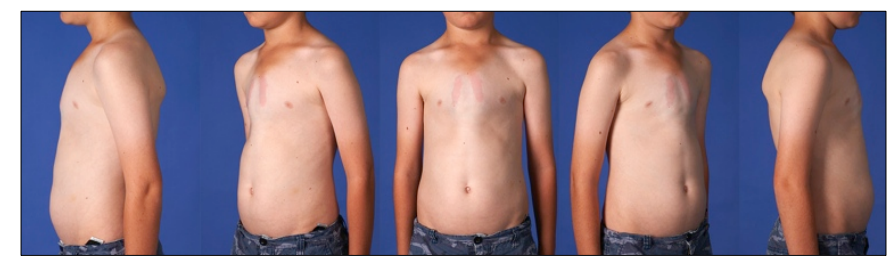

Figure 3b. Arms are by side.

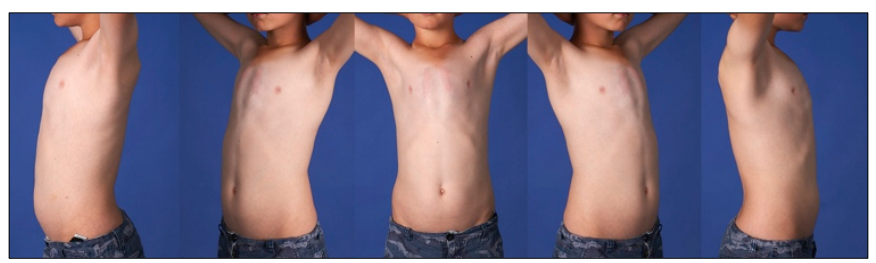

Figure 3c. Arms are raised. 
The patients are asked to inhale, then to exhale at which point the image is captured. Although the significance of the pectus protrusion is minimized during expiration a degree of standardization is ensured.

At the request of our institution's managing orthotist, the mark/impression visible at the point of contact of the brace with the apex of the pectus (the prominence) is captured in the photography to ensure that a record exists of whether the brace is fitted in the correct position.

The field of view extends from the patient's chin to the patient's waist. Patients and their families are informed about this in order to reassure them that the images are nonidentifiable. Male patients are photographed with their clothing above the waist removed while female patients, (the minority of the cohort in our institution), are offered alternatives. Female patients who consent to being photographed may choose to be photographed in their underwear, or with a top garment provided by our department. Depending on the location of the pectus on the chest wall, clothing for female patients can be adjusted to demonstrate the area affected and female patients can utilize the department's change room as required.

\section{Image Processing}

All images are captured in RAW format with copies of the unedited images stored electronically along with a copy of the signed consent form for the photographic session. Capture One (Phase One) software is utilized to make the following adjustments:

- White balance is corrected to the predetermined studio values

- Images are cropped to a $5 \times 7$ aspect ratio to ensure consistency of the field of view

- Processing and output results in $5 \times 7$ inch Tiff files at 300ppi.

All processed images are stored electronically.

The session images are collated via Adobe Photoshop to produce a single 5-image sequence (without the posterior view) as shown in Figure 3a, b, c, per page. This page is then available for printing for the purposes of on-going clinical care and treatment planning as well as to show to the patient at a subsequent clinical visit.

\section{D Photography}

Following the conventional (2D) photographic session, the patients are photographed using a 3D camera imaging system (3dMD). The $3 \mathrm{dMD}$ camera at The Children's Hospital at Westmead, Sydney consists of a standard five-pod camera installation. Four cameras are located in each corner of a supporting frame while a fifth camera is positioned directly above the patient in the center of the frame (Figure 4).

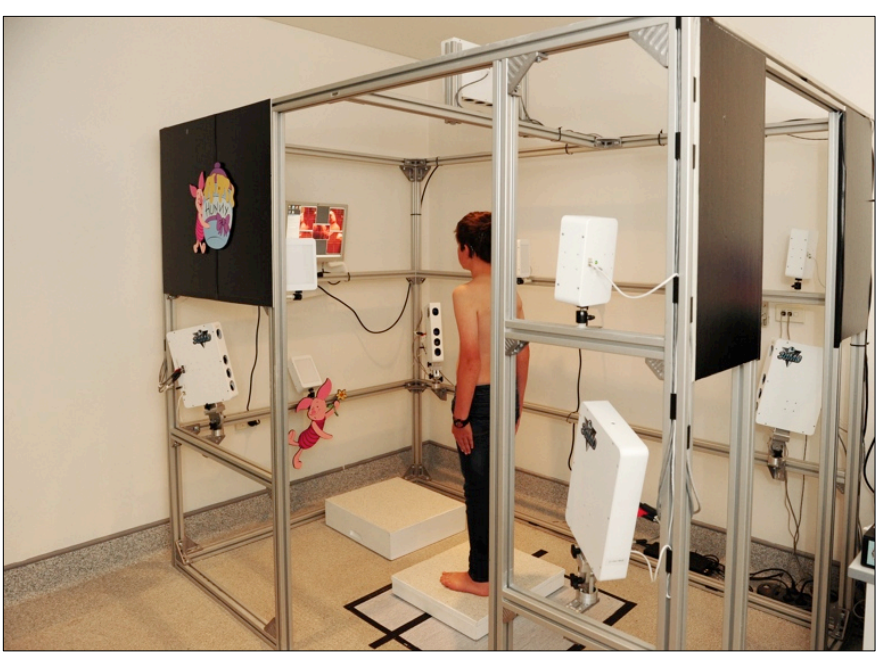

Figure 4. 3dMD Image capture.

A computer monitor is located at one end of the room, in front of the patient, which allows the patient to view their image during acquisition. This assists with ensuring correct positioning.

Although the technology of this imaging system allows for surface area and/or volume measurements to be made for objective evaluation, (Figure 5) the primary use of the imaging for patients with pectus carinatum is to track changes in the shape and appearance of the chest wall over time in order to provide visual, subjective feedback to the patient and their family during their clinical appointments.

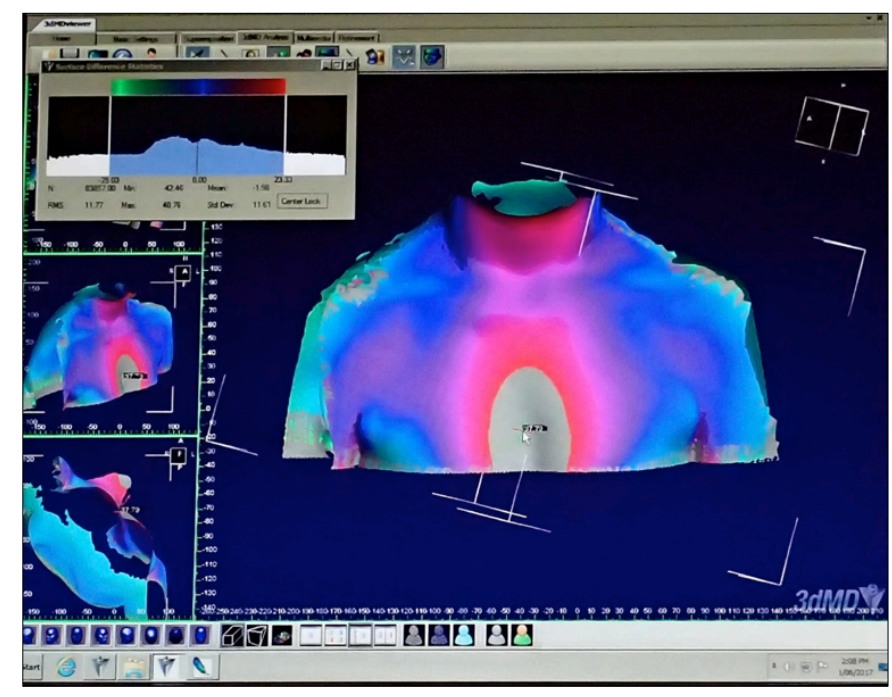

Figure 5. This image is formed by the $3 \mathrm{D}$ program when the images from the first and last visits are registered against each other. Image construction and calculation by Paul De Sensi.

The beige colored area shown in Figure 5 represents the area of change between a patient's two visits, approximately 15 months apart. For this patient, it was calculated that the volume minimized due to decompression of the pectus equates to 35 cubic $\mathrm{cm}$. 


\section{Discussion}

The Clinical Photography Department's involvement in the care of patients with pectus carinatum includes contributions that apply both directly and indirectly with patient care.

By encouraging the patient and his/her family to view their images at the time of acquisition, and to compare images with previous sessions the patients have an opportunity to selfevaluate their progress and receive immediate feedback from the orthotist. During clinical photography sessions, patients are often shown their unedited imaging from a number of views or aspects.

It is the practice of our department to process one of the $3 \mathrm{~d}$ images immediately in the presence of the patient for demonstration purposes. The patients and their families are generally quite intrigued by the quality and rotation ability of the 3D image. (Figure 6)

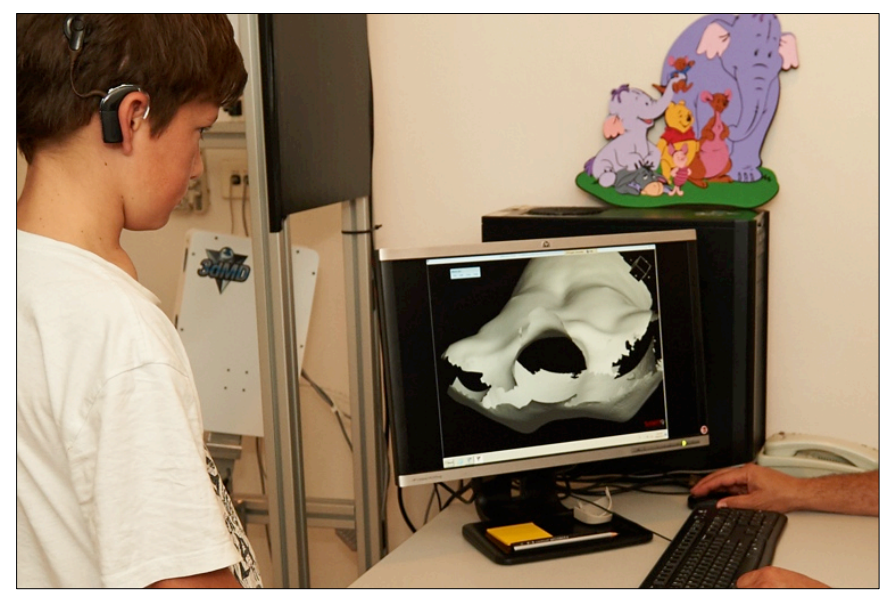

Figure 6. Patient viewing a newly captured 3D image.

The 3dMD imaging software allows for an image of a previous visit to be viewed alongside the current image. (Figure 7)
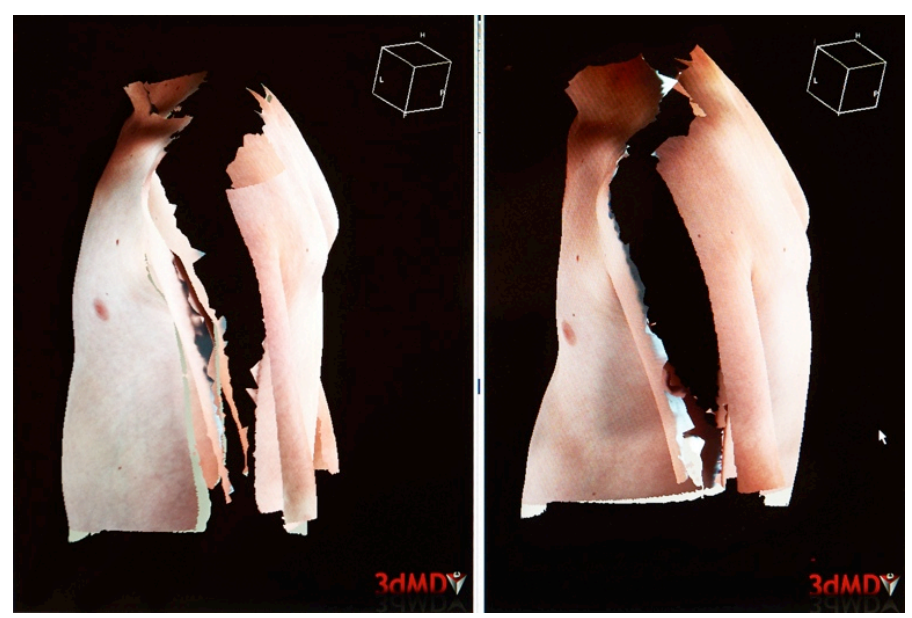

Figure 7. Demonstration of skin mode with left lateral view. The pretreatment is on the left. The image on the right is post 12 months' treatment.
A 'toggle texture' is the preferred viewing mode as it facilitates viewing contours of the thoracic area in comparison with 'skin mode'. (Figure 8)
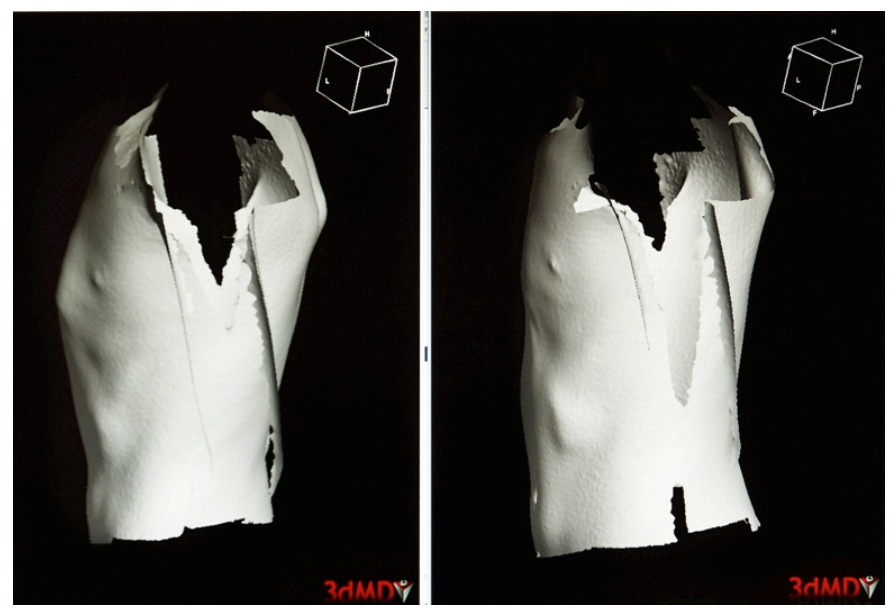

Figure 8. 3dMD Toggle Texture mode. The image on the right was taken 1 month following the one on the left. Quite a significant change in the chest wall can be noted following application of brace.

On occasions where there has been a realized lack of improvement, the demonstration of these images almost always leads to encouragement and the creation of renewed goals and resolutions on the part of the patient and family, in conjunction with further positive reinforcement by the orthotist.

In addition to this direct contribution to patient care (the standardized nature of the clinical photography) both conventional and 3D, creates several indirect opportunities for enhancing patient care. Changes in the chest wall shape can only ever be truly appreciated in the context of standardized imaging sequences and views, and adherence to a standardized protocol leaves our health professionals well placed to compare the effects of different treatments over time both within and between groups of patients.

\section{Conclusion}

The protocol and interactions described in this paper demonstrate the clinical utility of the Clinical Photography Department as an active contributor to patient care. While clinical record keeping is certainly a primary function of medical photographers, as a profession we are in a unique position to provide a standardized, professional, high quality service to our patients that can directly influence treatment outcomes. In the case of the management of patients with pectus carinatum, the clinical photography department at The Children's Hospital at Westmead now serves as an integral part of the patient's overall treatment plan with our photography and our interactions providing valuable 
information and incentive for the patient and their family to persevere with wearing their specific Orthotic appliance. This greatly assists our patients' psychological well-being.

Undoubtedly the most influential factor in ensuring that medical photography is relevant and vital to the management of conditions such as pectus carinatum is the effective liaison and communication that exists with the relevant clinical departments and their staff. Clinical photographers have a unique set of skills and should not be viewed (either internally or externally) as simply 'technicians'. In an era in which medical photographic departments are gradually being made redundant in many hospitals, involvement in clinical procedures and case management as described in this paper will assist to ensure that clinical photographers maintain their relevance and viability, and contribute to successful clinical outcomes as active members of healthcare teams.

\section{Acknowledgements}

The author wishes to thank the following for their help in gathering of information for this article:

Sean Hogan - Department Head, Orthotics Department at The Children's Hospital at Westmead, Sydney, NSW Australia

Paul De Sensi - Chief Medical Photographer at The Children's Hospital at Westmead, Sydney, NSW Australia

David Fitzsimons - Speech Pathologist, at The Children's Hospital at Westmead, Sydney, NSW Australia

The author would also like to thank the Biocommunications Association Endowed Fund for Education (EFFE) for helping fund the presentation of this paper at Biocomm2017 Conference.

\section{References}

Krummel TM. 1998. Congenital Malformations of the Lower Respiratory Tract in Children. Sixth Edition, Philadelphia: W.B. Saunders pp. 287.

Nuchtern JG, Mayer OH, Redding G, Hoppin AG. 2012.

Pectus carinatum. UpToDate, Waltham, 2012

https://www.uptodate.com/contents/pectus-carinatum

Goretsky MJ, Kelly RE Jr, Croitoru D, Nuss D. 2004. Chest wall anomalies: pectus excavatum and pectus carinatum. Adolesc. Med, Vol. 15. pp. 455-171.

Hefti, F. 2007. Thoracic Deformities. In Pediatric Orthopaedics in Practice. Berlin: Springer - Verlag pp. 122.
Koumbourlis AC. 2015. Pectus Deformities and their Impact on Pulmonary Physiology. Paediatric Respiratory Reviews, Vol. 16. Pp. 18-24.

\section{Author}

John Yeats, B.V.Sc., is a Registered Biological Illustrator (AIMBI) and has worked as a medical photographer in the Clinical Photography Department at The Children's Hospital at Westmead, Sydney Australia for 10 years.

Email: john.yeats@health.nsw.gov.au

\section{Licensing}

The author has chosen to license this content under a Creative Commons Attribution, NonCommercial, NoDerivatives 4.0 International License.

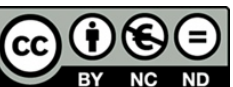

